\title{
Self-consistent scheme for toughness homogenization
}

\author{
Stéphane Roux * \\ LMT-Cachan \\ ENS de Cachan / CNRS-UMR 8535 / Université Paris 6 / UniverSud Paris \\ 61 avenue du Président Wilson, F-94235 Cachan Cedex, France.
}

(stephane.roux@lmt.ens-cachan.fr)

\section{François Hild}

LMT-Cachan

ENS de Cachan / CNRS-UMR 8535 / Université Paris 6 / UniverSud Paris

61 avenue du Président Wilson, F-94235 Cachan Cedex, France.

(hild@lmt.ens-cachan.fr)

\begin{abstract}
Considering a semi-infinite planar crack propagating along a plane where the local toughness is a random field, the addressed problem is to compute the effective (or homogeneous and macroscopic) toughness. After a brief introduction to the two regimes - strong and weak pinning - that are expected depending on the system size, a self-consistent homogenization scheme is introduced. It is shown that this scheme allows one to predict not only the mean value but also the standard deviation and even the complete probability distribution function of the toughness. A discussion about the quality of this prediction as compared with direct numerical simulations is proposed.
\end{abstract}

Keywords: Brittle fracture, Random toughness, Homogenization, Self-consistent scheme.

\footnotetext{
* to whom correspondence should be addressed. Fax: +33147402240.

(c) 2008 Kluwer Academic Publishers. Printed in the Netherlands.
} 


\section{Introduction}

Homogenization of heterogeneous materials has been a very active domain in the last decades [1]. The underlying philosophy is to note that many mechanical properties of heterogeneous solids do not require an exhaustive description of the microstructure and can be compared to those of "equivalent" homogeneous solids (e.g., representative volume elements). This observation concerns in particular elasticity. Non-linear constitutive laws such as plasticity are typically more sensitive to the details of the microstructure and yet significant progress has been achieved in the past years [2].

Amazingly, toughness homogenization has not received much attention, although the problem may be formulated in similar terms. In contrast to crack initiation, which is usually controlled by material defects or surface flaws (and hence not amenable to homogenization) [3], steady state crack propagation even in random fields of local toughness leads to self-averaging and allows one to define an "equivalent" (i.e., homogeneous and macroscopic) toughness.

However, in contrast to standard homogenization problems, an interesting partition in two regimes is observed, depending on the crack front length [4]. The "weak pinning" regime expected only for small system sizes turns out to be a simple problem leading to trivial results. Conversely, in the strong pinning regime, a multiplicity of equilibrium configurations appears, whose selection will be dictated by the temporal evolution. This specific feature turns the problem into a challenging theoretical issue. It has been shown that the onset of crack propagation is 
actually a genuine second order phase transition - called "depinning"

— that is observed in a wide range of different physical problems [5-7].

One consequence of this property is that all homogenization techniques are expected to fail. In a few words, the argument is that, even though no characteristic scale exists at a critical point and correlations extend to infinity, homogenization most often ignores correlations above a microscopic size and hence cannot do justice to the very nature of the physical phenomenon at play.

After defining the problem, the nature of the "weak" and "strong" pinning regimes will be recalled in Section 2. Then, in Section 3, the self-consistent approximation methodology is introduced. The resulting analysis is carried out up to the complete expression of the probability distribution function (p.d.f.) of the macroscopic toughness, as presented in Section 4. The validity of these results when compared with direct numerical simulations will be discussed in Section 5 .

\section{Problem definition}

Let us consider a mode I planar crack propagating along the $y$ direction, and whose axis is parallel to the $x$ direction as shown in Figure 1. The crack is assumed to remain within the plane $z=0$ where the local toughness is assumed to be random, $K_{c}(x, y)$. The surrounding medium (on both sides of the weak plane) is assumed to be a homogeneous elastic solid $[8,9]$. 
The local toughness is a random variable $K_{c}(x, y)$. It is characterized here simply by its p.d.f. and its correlation length here assumed to be finite. Moreover, for the sake of generality, one distinguishes the correlation length along the propagation direction, $\xi_{y}$, and along the crack front $\xi_{x}$. In the sequel a perturbation expansion valid only to first order is used, and hence, the local toughness is assumed to have a narrow distribution. In all physical cases, the maximum toughness is bounded, and hence the toughness is normalized with respect to its maximum value $K_{c}$ max. A dimensionless local toughness $\kappa_{c}$ as $\left(K_{c} \max -K_{c}\right) / K_{c} \max =\epsilon \kappa_{c}$ is introduced, where $\epsilon$ is the small parameter of the perturbation expansion, so that $\kappa_{c}$ is a positive random variable of order one (beware of the fact that the higher the toughness, the lower the $\left.\kappa_{c}\right)$. The p.d.f. of $\kappa_{c}$ is denoted by $p\left(\kappa_{c}\right)$.

It is noteworthy that this problem has been addressed in the literature in connection with dynamic crack propagation [10-12], however not under the viewpoint of homogenization. In the sequel, we will essentially focus on the quasi-static regime.

The crack propagation is most often characterized by a constitutive law relating the stress intensity factor (or energy release rate) to the crack velocity [3]. However, disregarding subcritical crack growth, there exists a critical stress intensity factor below which the crack velocity is strictly zero. In the sequel, we will not be interested in the propagation regime that will depend on the constitutive law of specific materials, but rather on the onset of crack propagation, that is only focus on the critical stress intensity factor. Thus the condition for crack arrest is simply that for all $x$, the local stress intensity factor is less than or 
equal to the local critical stress intensity factor

$$
K(x) \leq K_{c}(x, y)
$$

where $K(x)$ denotes the stress intensity factor at the point $(x, y=h(x))$ along the front $y=h(x)$.

The whole difficulty of the problem is that the front will not keep a rectilinear geometry as the crack advances. Rather it will roughen to conform to the facing toughness. Hence the geometry of the crack front, which is described by a function $y=h(x)$, has to be accounted for. Because of this meandering, the local stress intensity factor will be modulated. However, as above mentioned, the heterogeneity is assumed to be small, and only a perturbation expansion in $\epsilon$ will be used. At a large distance from the crack front, the roughening of the crack front can be neglected. This is how the "equivalent" homogeneous toughness may be introduced, namely, for the homogeneous solid, let us introduce $K_{0}$ as being the stress intensity produced by the remote loading. As the crack front is straight in that case, the macroscopic and microscopic stress intensity factors are identical. For the heterogeneous case, with the same mean location of the crack front, the stress intensity factor is modulated by the crack front geometry. Gao and Rice [13] showed that up to first order in perturbation, the stress intensity factor is written as

$$
K(x)=K_{0}\left[1+\frac{1}{2 \pi} \int \frac{h\left(x^{\prime}\right)-h(x)}{\left(x^{\prime}-x\right)^{2}} \mathrm{~d} x^{\prime}+\text { h.o.t. }\right]
$$

Thus the relative correction term, $k \equiv\left(K_{0}-K(x)\right) /\left(\epsilon K_{0}\right)$, is expressed as the convolution of the crack front shape $h(x)$ by a kernel $H(x)=-x^{-2}$. Following the definition of $\epsilon$, at zeroth order in this 
small parameter, one simply has $K_{0}=K_{c} \max$. Thus at first order in $\epsilon$, the arrest condition reads

$$
k(x)>\kappa_{c}(x, h(x))
$$

\subsection{Solution}

The convolution with the kernel $H(x)$ may be inverted to provide the Green's function $G(x)$, leading to the determination of the crack front shape $h(x)$ for a given $k(x)$ profile

$$
h(x)=h_{0}+G(x) \star k(x)
$$

where the $\star$ denotes a convolution. However, $G(x)$ displays a logarithmic variation with $x$, and hence the cut-offs at small and large length scales play a key role. The small size cut-off requires the explicit introduction of the correlation length $\xi_{x}$ so that one can smear out the local divergence through an integration of stress intensity factor over this correlation length. However, the divergence of the Green's function at large distances signals that the crack front length, $L$, or more precisely the ratio $L / \xi_{x}$, is an explicit cut-off that will give rise to size effects.

Let us consider the simple case where the arrest condition is an exact equality at all points along the front,

$$
k(x)=\kappa_{c}(x, h(x))
$$

In that case, the crack front shape obeys the non linear equation

$$
h(x)=h_{0}+G(x) \star \kappa_{c}(x, h(x))
$$


For each $h_{0}$ (corresponding to progressively driving the crack through the random field), one would like to compute $h(x)$, which is however a difficult task in the most general case.

\subsection{WEAK PINNING}

If $\kappa_{c}(x, y)$ varies slowly with $y$ (an extreme case being the layered case where $\kappa_{c}$ only depends on $x, \partial \kappa_{c} / \partial y=0$ ), then it is easy to solve for Eq. (6), since the non-linearity is "weak" and can be treated perturbatively (for the layered case, the non-linear term vanishes, and the problem becomes linear). This case will be referred to as "weak pinning."

In that case, because the stress intensity factor at each point matches the local toughness, averaging Eq. (6) along $x$ leads to the determination of the macroscopic critical stress intensity factor, $K_{c}$ hom, as being equal to the arithmetic average of the local toughness

$$
K_{c h o m}=\left\langle K_{c}\right\rangle
$$

Let us underline that a "weak" non-linearity does not affect the result. It should also be noted that only a first order perturbative treatment in the disorder is used, and hence, arithmetic, geometric or harmonic averages are equivalent, and higher order terms should be used to distinguish between them.

The above statement has a straightforward energetic interpretation. Again, because of the first order perturbation treatment that is proposed herein, relative stress intensity factors, or relative energy release rates are identical (up to a trivial factor 2). Hence the above result, 
Eq. (7), is retrieved balancing the macroscopic estimate of the critical energy release rate and the energy cost obtained from the integration of the local surface energy.

The key question is to understand the limit of validity of this weak pinning regime. In fact, the break-down of the above argument comes from situations where $\partial h(x) / \partial h_{0}<0$. Such a situation appears in a number of different situations such as the celebrated Kardar-ParisiZhang equation [14], or the underlying Burgers equation. In that case, many solutions for the crack front conformation exist for which the arrest condition cannot be fulfilled as an equality at every point. This causes the front to jump from one configuration to the next one through a series of instabilities.

\subsection{Strong PINNing}

The interesting observation is that the divergence the Green's function with the crack front length $L$ imposes that for any given disorder (for which $\xi_{y}$ is finite), there exists a length above which weak pinning is no longer ensured. Thus, asymptotically (i.e., for large front lengths), the relevant regime is "strong pinning."

The occurrence of micro-instabilities has far reaching consequences. In particular, the argument leading to the macroscopic toughness as being the average of the local ones no longer applies. Whatever the speed at which the fracture front is driven, the front will locally "depin" from an equilibrium conformation and jump to the next one at a rate that cannot be controlled at the macroscopic scale. 
Although we did not specify the dynamic equation that describes these transitions, it will dissipate energy either locally (by visco-elasticity or plasticity) or remotely as sound waves are radiated and dampened by any possible internal friction mechanism. The consequence is that the macroscopic toughness is greater than the average local toughness, the difference between these two quantities being the additional dissipation occurring in micro-instabilities.

Unfortunately, it is difficult to solve exactly the random non-linear equation (6). Hence, we propose to address this question from a simple prescription that has revealed extremely useful for other homogenization problems, namely, the self-consistent approximation [4]. It is an approximation, and it will not exhaust the full complexity of the problem. However, it allows one to reproduce the transition from weak to strong pinning regimes, and to provide a quantitative criterion. It will also lead to predictions concerning not only the mean macroscopic toughness of finite size systems, but also on the entire p.d.f. of the macroscopic toughness.

\section{Self-consistent scheme}

Let us consider a slice of the random toughness field of width $\xi_{x}$ and infinite length. Away from this strip $|x|<\xi_{x} / 2$, one substitutes to the random toughness a homogeneous one, $K_{c}$ hom as shown in Figure 2. Yet at this stage, $K_{c}$ hom is undetermined. The spirit of the selfconsistent approximation [15] is that if this substitution is "neutral," 
then the macroscopic toughness is equal to $K_{c \text { hom. Neutrality means }}$ that, on average, the front remains flat $\langle h(L / 2)-h(0)\rangle=0$. This latter requirement is the natural application of the self-consistency concept used for homogenization.

Since away from the strip, the medium is homogeneous, the crack front geometry is directly read from the Green's function. As mentioned previously, the divergence of the Green's function at large distance prompts us to use a finite width medium, $-L / 2<x<L / 2$ (in numerical simulations, periodic boundary conditions are the most appropriate way to deal with finite size effects). The Green function can be obtained from the inverse Fourier transform of $1 / \widetilde{G}(k)$. Hence, since $\widetilde{G}(k) \propto|k|$, we have

$$
h(0)-h(L / 2)=A \log \left(L / \xi_{x}\right)\left(\kappa_{c}(x=0, h(0))-\kappa_{c} \text { hom }\right)
$$

The proportionality constant is not specified since it depends on the chosen prescription for introducing finite boundary conditions, but the most meaningful dependencies are retained. Solutions of $h(0)$ are visualized as the intersection between a known random profile in $y$ $\kappa_{c}(x=0, y)$ and a linear relation $\kappa_{c \text { hom }}-(y-h(L / 2)) /\left[A \log \left(L / \xi_{x}\right)\right]$ as shown in Figure 3. It is possible to recover from this construction the previous discussion on weak and strong pinning by counting the number of possible solutions. It is to be noted that the slope $S$ of the straight line scales as $1 / \log \left(L / \xi_{x}\right)$. Hence, as the front length increases, a transition from weak to strong pinning will occur as the above slope reaches the maximum slope $\partial \kappa_{c}(0, y) / \partial y$. 
The macroscopic toughness is again given by the average of the local toughness, with however one major restriction, namely, the average is to be taken over values that are visited as the crack moves onward. As shown in Figure 4, the $\kappa_{c}$ values that are averaged over are the most salient ones, whereas the smallest do not correspond to visited equilibrium configurations. As noted previously, this phenomenon indicates that the macroscopic toughness will be greater than the average value of the local toughness. This self-consistent procedure has been introduced and discussed in Ref. [4], and hence the reader is referred to this reference for a more complete discussion on the main results concerning the macroscopic toughness.

\section{Probability distribution function of the macroscopic toughness}

The probability that a specific value $\kappa_{c}$ correspond to a visited stable configuration is written as $r\left(\kappa_{c}\right)=B p\left(\kappa_{c}\right) Q\left(\kappa_{c}\right)$, where $Q\left(\kappa_{c}\right)$ is the probability that no previously encountered value of the toughness may have screened the present site, and $B$ a multiplicative constant $(1 / B=$ $\left.\int p(x) Q(x) \mathrm{d} x\right)$. Note that $r(\kappa)$ is exactly the p.d.f. of the macroscopic toughness. Indeed, the missed equilibrium configurations are unstable and cannot be observed.

Let $P(x)$ be the cumulative distribution of local toughness 
, $P(x)=\int_{0}^{x} p(x) \mathrm{d} x$. For a given slope $S=\left(A \log \left(L / \xi_{x}\right)\right)^{-1}$, one may write

$$
Q(\kappa)=\prod_{i=1}^{\infty}\left(1-P\left(\kappa-i S \xi_{y}\right)\right)
$$

In the limit where $S \xi_{y}$ is a small parameter as compared to the width of the distribution $p$, the above discrete product is approached as its continuum limit

$$
Q(\kappa)=\exp \left[\frac{1}{S \xi_{y}} \int_{0}^{\kappa} \log (1-P(x)) \mathrm{d} x\right]
$$

Let us consider the example of a local toughness distribution that is uniform over the interval $[0 ; 1]$. In that case, $P(\kappa)=\kappa$, and hence

$$
Q(\kappa)=\exp \left[-\frac{(1-\kappa) \log (1-\kappa)+\kappa}{S \xi_{y}}\right]
$$

The interesting limit is that of a large system size where $S \rightarrow 0$. By performing a Taylor expansion about the origin where $Q$ will assume non negligible values, $(1-\kappa) \log (1-\kappa)+\kappa \sim(1 / 2) \kappa^{2}$ and hence

$$
Q(\kappa) \sim \exp \left[-\frac{\kappa^{2}}{2 S \xi_{y}}\right]
$$

so that $Q$ is seen as a filter for the distribution $p(x)$ preserving large toughnesses (small $\kappa$ ), and cutting out $\kappa$ larger than $\kappa^{*} \sim \sqrt{S \xi_{y}}$. Rescaling $\kappa$ by $\kappa^{*}$ provides a scale-independent distribution

$$
Q(\kappa)=\Psi_{1}\left(\kappa / \kappa^{*}\right)
$$

where

$$
\Psi_{1}(x)=\exp \left(-x^{2} / 2\right)
$$

and $\kappa^{*}$ gathers all the size dependence given by the $S$ factor, and hence

$$
\kappa^{*} \sim \xi_{y}^{1 / 2} \log ^{-1 / 2}\left(L / \xi_{x}\right)
$$


Figure 5 illustrates this property, showing a series of p.d.f.s for different system sizes (or equivalently $S$ ), and their plot as functions of $\kappa / \kappa^{*}$. Similarly, Figure 6 is a direct check of the scaling of $\kappa^{*}$ as a function of $S^{1 / 2}$ and a linear behavior is observed.

It is of interest to note that the resulting distribution $\Psi$ is the same for all distributions $p$ such that $p(0)$ is finite. Hence, the result has a rather wide degree of generality that may not have been anticipated. However, not all distributions $p$ belong to this category. If $P(x) \sim_{x=0}$ $x^{\beta}$, then it is again observed that for large $L$, the $Q$ distribution takes a unique form

$$
Q(\kappa)=\Psi_{\beta}\left(\kappa / \kappa^{*}\right)
$$

where

$$
\Psi_{\beta}(x)=\exp \left[-\frac{x^{1+\beta}}{(1+\beta)}\right]
$$

and

$$
\kappa^{*} \sim \xi_{y}^{1 /(1+\beta)} \log ^{-1 /(1+\beta)}\left(L / \xi_{x}\right)
$$

For all values of $\beta$ a different asymptotic distribution emerges. Thus a single scalar characterizes this asymptotic behavior, namely the shape of the distribution of local toughness close to its maximum. The exponent $\beta$ is defined without many restrictions as

$$
\beta=\lim _{x \rightarrow 0^{+}} \frac{\log (P(x))}{\log (x)}
$$

and finally the effective p.d.f. of the macroscopic toughness, $r(\kappa)$, assumes the form $R_{\beta}\left(\kappa / \kappa^{*}\right)$ where

$$
R_{\beta}(x)=x^{\beta} \exp \left[-\frac{x^{1+\beta}}{(1+\beta)}\right]
$$


It is interesting to note that in a different context, namely damage mechanics, a systematic classification of the expected size effects was already argued by Hansen et al. [16] to be controlled by such quantities as the one defined in Eq. (19) on the basis of scale invariance.

From this expression it is possible to argue that the mean macroscopic toughness displays the systematic size effect as any other moment, namely,

$$
\left\langle\kappa_{c} \text { hom }\right\rangle \sim \xi_{y}^{1 /(1+\beta)} \log ^{-1 /(1+\beta)}\left(L / \xi_{x}\right)
$$

(Note that this result holds for the "reduced" expression $\kappa$, so that $K_{c}$ hom tends to a constant (i.e., the maximum local toughness) plus the above expression that appears as a very weak size effect).

\section{Discussion}

As mentioned in the introduction, the very nature of the onset of crack propagation has been claimed to be a genuine 2nd order phase transition, and hence, ignoring correlations above a microscopic scale is an oversimplification. Hence the self-consistent approach is deemed to fail a priori. However, it is interesting to investigate the limits of the approach and its transposition to a regime where the occurrence of micro-instabilities is a major difficulty as compared with traditional homogenization problems.

This section lists some of the properties that have been observed in numerical studies of the problem in the strong pinning regime as reported in Ref. [17]: 
- First, the mean macroscopic toughness $\left\langle K_{c}\right\rangle$ tends to a constant $K_{\infty}$ for large system sizes, as reproduced from the self-consistent approximation.

- The standard deviation of the macroscopic toughness $\sigma\left(K_{c}\right)$ vanishes as a universal power-law of the front length. Qualitatively, a size effect law is observed for this standard deviation in the selfconsistent scheme, but in quantitative terms it appears to be a much weaker effect (typically a power-law of the logarithm of $L$ ).

- In order to define the local toughness distribution, an infinite number of parameters is needed. At a macroscopic level, we observe that the number of parameters needed to characterize the effective toughness distribution has been significantly reduced. The self-consistent approach predicts that only three parameters are needed, namely one for the thermodynamic limit $K_{c \infty}$, one for the width of the distribution $\sigma\left(K_{c}\right)$, and one for the exponent $\beta$. However, in direct simulations, it is observed that only the two former parameters are needed. All values of exponent $\beta$ defined at the microscopic level seem to lead to the same unique form for the normalized macroscopic toughness, $\left(K_{c \infty}-K_{c}\right) / \sigma\left(K_{c}\right)$. Thus, although the trend is correctly reproduced, the local nature of the self-consistent scheme miss part of the universality of the complete model.

Thus it appears that, to a certain degree, the self-consistent approach reveals a robustness of the asymptotic (i.e., large $L$ ) behavior. The latter is however not as strong as in direct numerical simulations 
where universality is observed. The reason for this partial success is presumably to be understood through the fact that the random toughness is preserved only along a line in the self-consistent prescription thereby overemphasizing the individual role played by the local toughness statistics. In direct simulations, depinning appears to be a more collective phenomenon, hence less sensitive to the details of $p$.

Let us finally observe that experimentally, the situation used to be quite confusing, as the predicted scaling of the crack front roughness exponent from numerical simulations did not seem to be obeyed [18]. However, quite recently, a more careful analysis of the data rather tends to support the strong pinning modeling above a small scale that is affected by the system preparation [19]. An extension of this theory to three dimensional cracks that allow for an experimental interpretation of 3D crack roughness from such a planar pinning problem has been recently proposed [20]. Last, still on the experimental side, let us mention that the analysis of crack arrest statistics of indentation cracks has been proposed based on the above presented depinning critical features [21].

\section{Conclusions}

Crack propagation in a random brittle material may be seen as a depinning phenomenon that displays two distinct regimes. A weak pinning, where the homogenization turns out to give a trivial result, namely the macroscopic equivalent toughness is nothing but the average of the local toughness. This regime is however restricted to small crack 
sizes or strongly anisotropic textures along the propagation direction. The second regime, strong pinning, relevant for most practical cases, consists in a series of micro-instabilities for which a homogenization approach is a priori more difficult to formulate.

It has been proposed to address this second regime within the socalled self-consistent approach. The latter turns out to reveal a wealth of information, not only for the mean macroscopic toughness but also its fluctuations, and even the entire probability density function for the macroscopic toughness. The prediction is that the re-scaled distribution of toughness tends towards one of an infinite series of stable distributions indexed by a single parameter. This result is however not as strong as the single universal distribution that is observed in direct numerical simulations, but is already quite a remarkable success, considering the fact that strong pinning is a second order phase transition where scale invariance is essential, and obviously violated in the self-consistent prescription.

\section{Acknowledgements}

We acknowledge useful discussions with Drs. A. Hansen and D. Vandembroucq. 


\section{References}

1. E. Sanchez-Palencia and A. Zaoui eds.: 1987, "Homogenization Techniques for Composite Media," Lecture Notes in Physics, vol. 272, (Springer, Berlin (Germany), 1987)

2. M. Bornert, T. Bretheau and P. Gilormini eds.: 2008, "Homogenization in Mechanics of Materials," (Lavoisier, Paris (France), 2008).

3. B. R. Lawn: 1993, "Fracture of Brittle Solids," (Cambridge University Press, Cambridge (UK), 1993).

4. S. Roux, D. Vandembroucq and F. Hild: 2003, "Effective toughness of heterogeneous brittle materials," Eur. J. Mech. A/SolidsVol. 22, pp. 743-749.

5. D. S. Fisher: 1985, "Sliding charge-density waves as a dynamic critical phenomenon," Phys. Rev. B Vol. 31 no. 3, pp. 1396-1427.

6. D. Ertas and M. Kardar: 1994, "Anisotropic scaling in depinning of a flux line," Phys. Rev. Lett.Vol. 73 no. 12, pp. 1703-1706.

7. J. P. Bouchaud, E. Bouchaud, G. Lapasset and J. Planes: 1993, "Models of fractal cracks," Phys. Rev. Lett.Vol. 71 no. 14, pp. 2240-2243.

8. J. Schmittbuhl, S. Roux, J.P. Vilotte and K. J. Måløy: 1995, "Interfacial crack pinning: Effect of nonlocal interactions," Phys. Rev. Lett.Vol. 74, no. 10, pp. 1787-1790.

9. A. Tanguy, M. Gounelle and S. Roux: 1998, "From individual to collective pinning: Effect of long-range elastic interactions," Phys. Rev. EVol. 58, no. 2, pp. 1577-1590.

10. G. Perrin and J. R. Rice: 1994, "Disordering of a dynamic planar crack front in a model elastic medium of randomly variable toughness," J. Mech. Phys. Sol.Vol. 42, no. 6, pp. 10471064.

11. S. Ramanathan and D. S. Fisher: 1998, "Onset of propagation of planar cracks in heterogeneous media," Phys. Rev. BVol. 58, no. 10, pp. 6026-6046.

12. J. W. Morrissey and J. R. Rice: 2000, "Perturbative simulations of crack front waves," J. Mech. Phys. Sol.Vol. 48, no. 6-7, pp. 1229-1251. 
13. H. Gao and J. R. Rice: 1989, "First-Order Perturbation Analysis of Crack Trapping by Arrays of Obstacles," ASME J. Appl. Mech.Vol. 56, pp. 828-836.

14. M. Kardar, G. Parisi and Y.C. Zhang: 1986, "Dynamic scaling of growing interfaces," Phys. Rev. Lett.Vol. 56 no. 9, pp. 889-892.

15. Hill, R.: 1965, "A Self-Consistent Mechanics of Composite Materials," J. Mech. Phys. Solids Vol. 13 pp. 213-222.

16. A. Hansen, E. L. Hinrichsen and S. Roux: 1991, "Scale-Invariant disorder in fracture and related breakdown phenomena," Phys. Rev. BVol. 43, pp. 665678.

17. D. Vandembroucq, R. Skoe and S. Roux: 2004, "Universal depinning force fluctuations of an elastic line: Application to finite temperature behavior," Phys. Rev. EVol. 70 no. 5, pp. 051101.

18. J. Schmittbuhl and K. J. Måløy: 1997, "Direct Observation of a Self-Affine Crack Propagation," Phys. Rev. Lett.Vol. 78 no. 20, pp. 3888-3891; A. Delaplace, J. Schmittbuhl and K. J. Måløy: 1999, "High resolution description of a crack front in a heterogeneous Plexiglas block," Phys. Rev. EVol. 60 no. 2, pp. 1337-1343.

19. S. Santucci: 2008, private communication.

20. D. Bonamy, L. Ponson, S. Prades, E. Bouchaud and C. Guillot: 2006, "Scaling exponents for fracture surfaces in homogeneous glass and glassy ceramics, " Phys. Rev. Lett.Vol. 97 no. 13, pp. 135504.

21. Y. Charles, D. Vandembroucq, F. Hild and S. Roux: 2004, "Materialindependent crack arrest statistics," J. Mech. Phys. Sol.Vol. 52, no. 7, pp. 1651-1669; Y. Charles, D. Vandembroucq, F. Hild and S. Roux: 2006, "Materials-independent crack arrest statistics: Application to indentation experiments," Int. J. Fract. Vol. 142 no. 1-2, pp. 51-67. 


\section{List of Figures}

1 Schematic plot of the crack front advancing through a random toughness field illustrated as a gray scale map.

2 Illustration of the self-consistent prescription. The full problem (left) is replaced by a homogenized version outside the strip. Imposing that the front remains flat determines the effective macroscopic toughness.

3 Construction of possible solutions of the crack front position along the $y$ axis. In the case of weak pinning, as shown on the left, a unique solution is obtained. In contrast, for strong pinning (shown on the right), several solutions appear, and hence the actual chosen solution as the crack is driven to the right is the leftmost solution. 24

4 Illustration of the time change of the crack front position at the origin as the front is driven. It is to be underlined that this motion consists of a succession of smooth change and abrupt discontinuities that are a signature of the strong pinning regime.

5 Raw (left) and Scaled (right) distribution of the macroscopic (and homogeneous) toughness for different values of the $S$ parameter. 
6 Change of the mean (macroscopic) toughness as a function of $S^{1 / 2}$ (for a unit correlation length $\xi_{y}=1$ ) in the case of a uniform local toughness distribution. Using $S \xi_{y}=A \xi_{y} \log \left(L / \xi_{x}\right)$, the above graph is an illustration of the very slow size effect that affects the macroscopic (and homogeneous) toughness. 


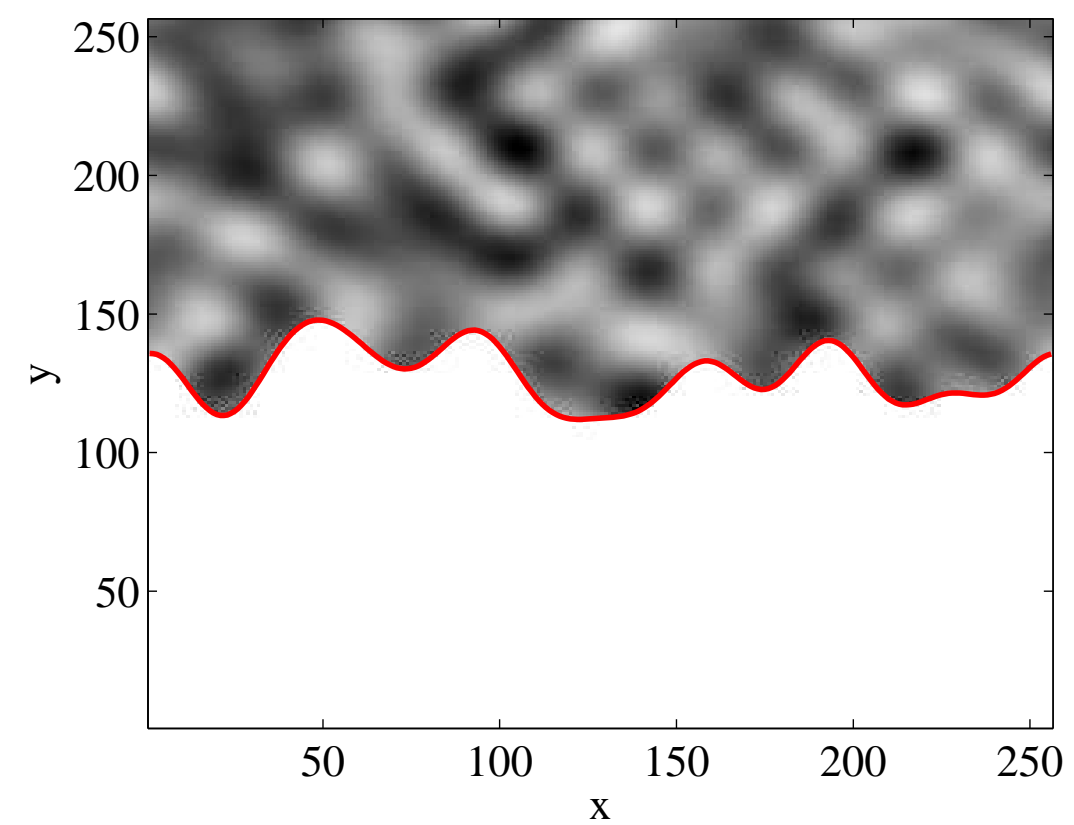

Figure 1. Schematic plot of the crack front advancing through a random toughness field illustrated as a gray scale map.

Roux et al. 


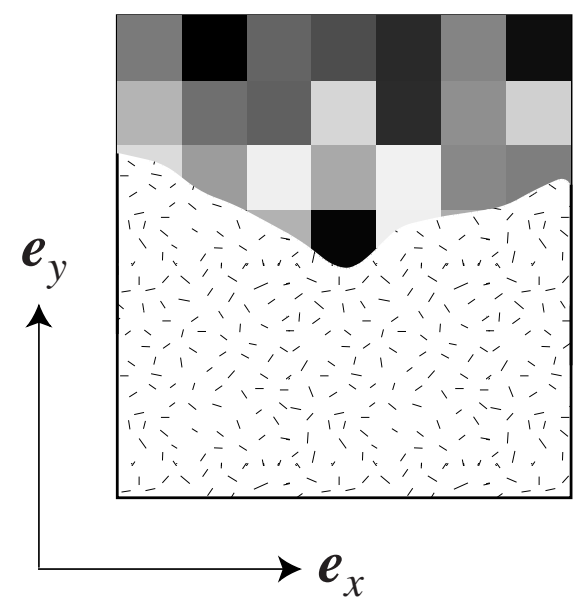

-a-

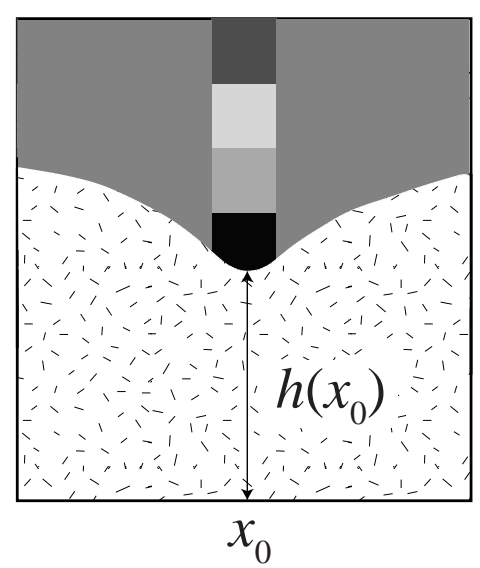

$-b-$

Figure 2. Illustration of the self-consistent prescription. The full problem (left) is replaced by a homogenized version outside the strip. Imposing that the front remains flat determines the effective macroscopic toughness.

Roux et al. 

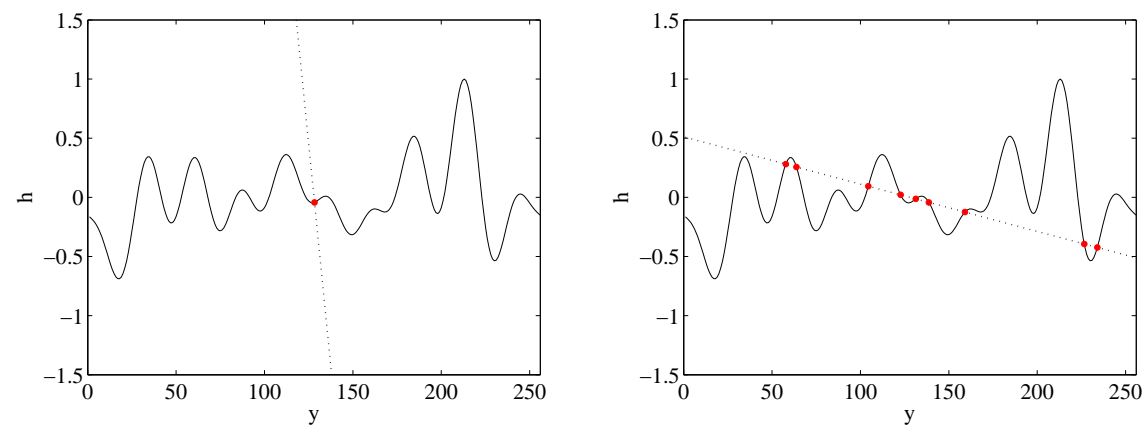

Figure 3. Construction of possible solutions of the crack front position along the $y$ axis. In the case of weak pinning, as shown on the left, a unique solution is obtained. In contrast, for strong pinning (shown on the right), several solutions appear, and hence the actual chosen solution as the crack is driven to the right is the leftmost solution.

\section{Roux et al.}




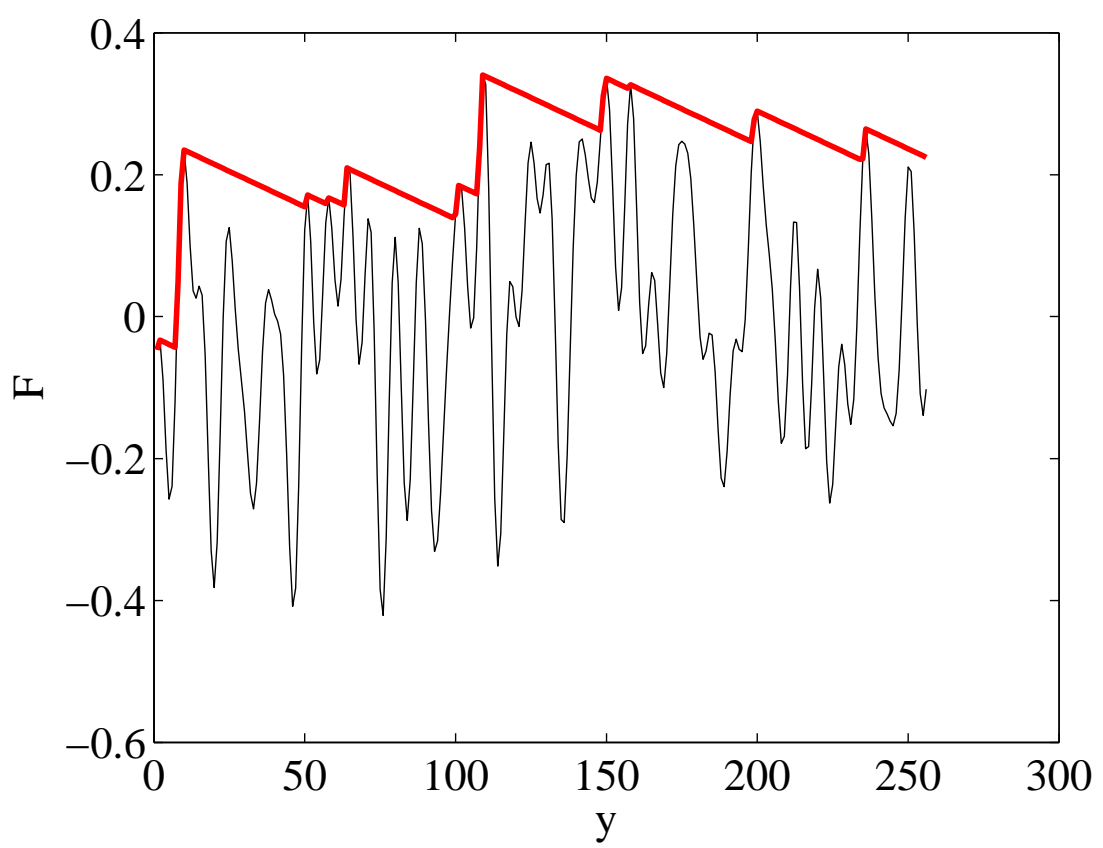

Figure 4. Illustration of the time change of the crack front position at the origin as the front is driven. It is to be underlined that this motion consists of a succession of smooth change and abrupt discontinuities that are a signature of the strong pinning regime.

Roux et al. 

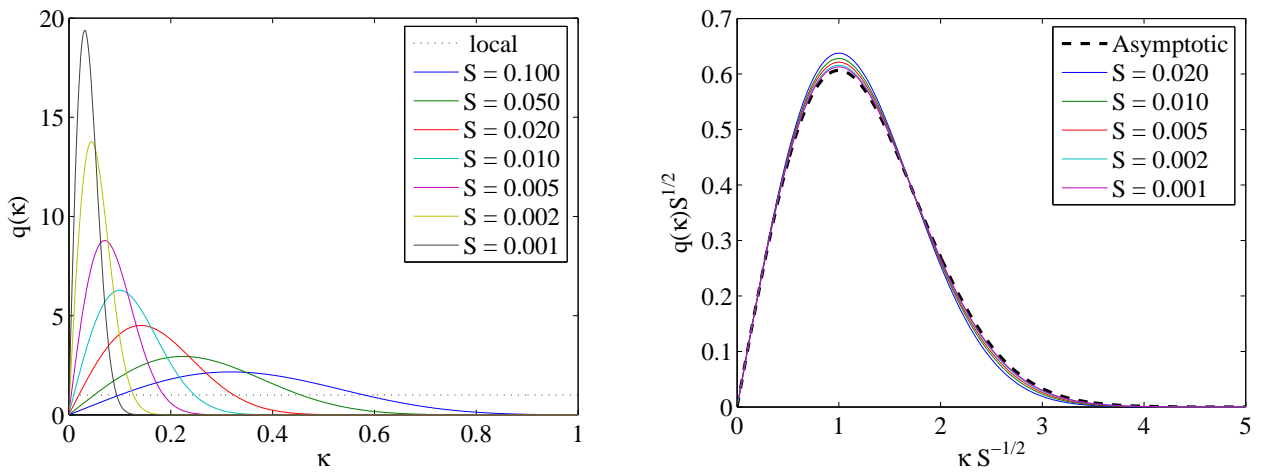

Figure 5. Raw (left) and Scaled (right) distribution of the macroscopic (and homogeneous) toughness for different values of the $S$ parameter.

Roux et al. 


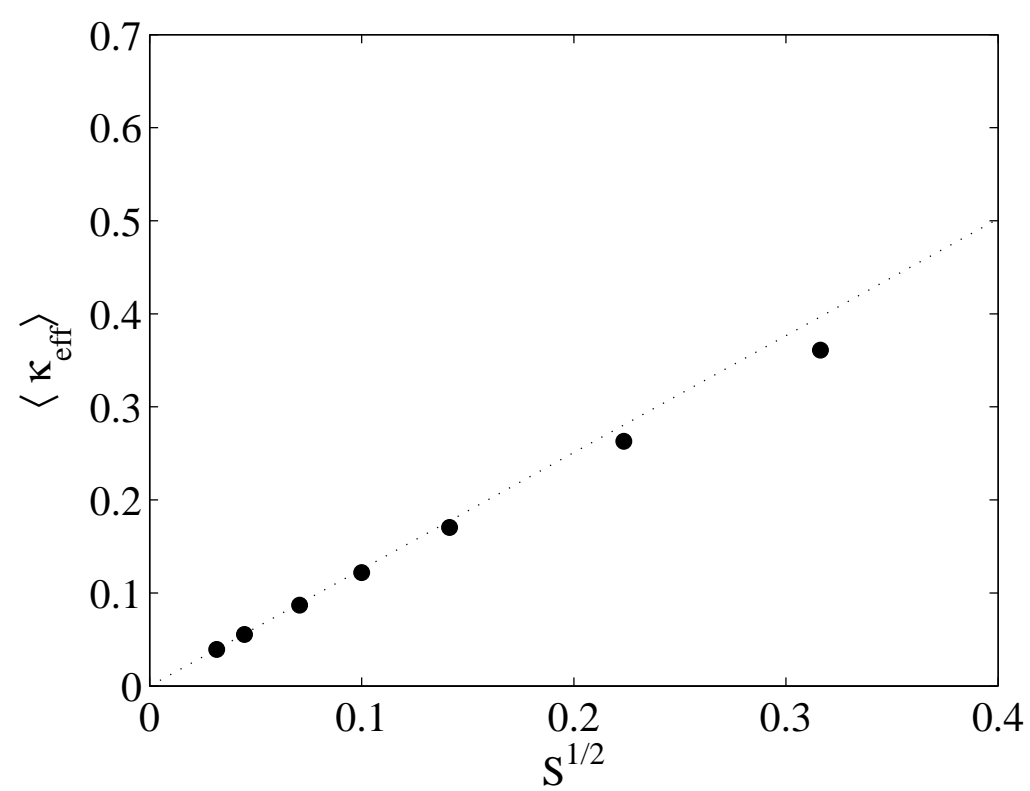

Figure 6. Change of the mean (macroscopic) toughness as a function of $S^{1 / 2}$ (for a unit correlation length $\xi_{y}=1$ ) in the case of a uniform local toughness distribution. Using $S \xi_{y}=A \xi_{y} \log \left(L / \xi_{x}\right)$, the above graph is an illustration of the very slow size effect that affects the macroscopic (and homogeneous) toughness.

Roux et al.

Address for Offprints:

Stéphane Roux (corresponding author)

LMT-Cachan, ENS de Cachan

61 avenue du Président Wilson, F-94235 Cachan Cedex, France. 
ijf08_rev.tex; 8/08/2008; 23:26; p.28 